\title{
ANALYZING MEASUREMENTS OF NONLINEAR TRANSFE FUNCTIONS WITH TSCHEBYSHEV POLYNOMIALS
}

\author{
G. A. Krafft, TJNAF, 12000 Jefferson Ave., Newport News, VA 23606
}

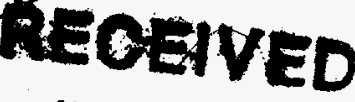
JUN 17 noon OS S T I

\section{Abstract}

Recently, due to advances in computers and data aquisition systems, the following type of measurement has become more common: (1) Impress a given modulation on a device to be tested. (2) Acquire a data stream, usually at equally spaced sample intervals, of the response of the system to the modulation. (3) Fit the data thereby acquired to some nonlinear function set that might (or might not!) describe the response of the device. In this paper it is pointed out that by choosing to modulate the test parameter sinusoidally, and by fast-Fourier transforming the acquired data stream, one unambiguously determines the Tschebyshev expansion of the response function around the working point, potentially yielding quantitative information about high nonlinear orders in the system response. The need for data fitting is thereby eliminated. A detailed example, the analysis of the nonlinear phase-phase transfer function in the Jefferson Lab injector, is presented.

\section{ANALYSIS}

The accelerator at Jefferson Lab acts like a LINAC in that the particle orbit never entirely closes on itself. Therefore, to analyse the performance of the accelerator, measurements of the beam transfer maps take on fundamental significance; there is no notion of tune as in a ring. In the course of analyzing such beam transfer maps, in particular the phase-phase transfer map between the chopping system and main linac in the injector, a convenient means was found for characterizing the system response, including nonlinearities that might exist in the transfer map. This type of analysis may be applied generally to measurements where one perturbs some parameter, e.g. the orbit around the working point of an accelerator, and measures the system response to that perturbation at the same time.

In general, consider a measurement where a parameter, $x$, is modulated between two limits, and simultaneously the response of the system under test is recorded. An interesting case is when the modulation is sinusoidal

$$
\delta x=\Delta x \cos (\omega t),
$$

where $\Delta x$ is the amplitude of the modulation and $\omega$ is the angular frequency of the modulation. For a given amplitude $\Delta x$, the response defines a function with dimensionless argument on the domain $[-1,+1]$ by $Y(\delta x / \Delta x)=$ $y(\delta x)$. If this function is expanded in an orthogonal series of Tschebyshev polynomials

$$
y(\delta x)=Y(\delta x / \Delta x)=\sum_{n=1}^{\infty} a_{n} T_{n}(\delta x / \Delta x),
$$

then using the standard identity defining Tschebyshev
polynomials, $T_{n}(\cos \theta)=\cos (n \theta)$, the amplitudes of the expansion coefficients appear directly as the size of the peaks in the Fourier analysis of the output data

$$
a_{n}=\frac{1}{\pi} \int_{0}^{2 \pi} Y(\cos \omega t) \cos (n \omega t) d(\omega t) .
$$

Examples of the types of distortions that correspond to two of the Tschebyshev modes are shown in Fig. 1. In these plots, the Tschebyshev modes are displayed, including a random noise component added to the ordinate, to model noise in the measurements, the peak to peak amplitude of the noise being one tenth of the mode amplitude. If the modulation is performed at a certain frequency $f=\omega / 2 \pi$, and the noise level is the same as appears in the mode pattern figures, then by Fourier analysis of the output of the phase detector, one obtains the power spectra in Figs. 2 and 3. Modulation in the $n=1$ mode gives output only at the first harmonic, modulation in the $n=2$ mode gives output only at the second harmonic, modulation in the $n=3$ mode gives output only at the third harmonic, and so forth. Conversely, if one sinusoidally modulates the input parameter and Fourier analyzes the output response around a particular working point, the response function is resolved into its Tschebyshev amplitudes, the expansion coefficient being the Fourier amplitude of the corresponding harmonic of the modulation frequency.

In Figs. 1, the total number of points is 1020 , and it is assumed for concreteness that the sample rate is $60 \mathrm{~Hz}$. A $1 \mathrm{~Hz}$ modulation was done, and it is clear that the signal to noise in the measurement is good in a total measurement time of $17 \mathrm{sec}$. It is also clear that this technique unambiguously establishes the response function, at least in a neighborhood of the working point, without the need of doing some form of nonlinear least fitting.

\section{MEASUREMENT SCHEME}

It has been known for many years that a good way to measure relative time-of-arrival of charged accelerator bunches is to use longitudinal pickup cavities and precision RF phase detectors. Such devices are used to set up, check, and optimize the bunch length of the bunches emerging from the Jefferson Lab injector [1]. In the "best" case, one could in principle compress the bunch length up to the limit given by the injected longitudinal emittance, the maximum permitted extracted energy spread, and Liouville's theorem on conservation of phase space area. In practice, nonlinearities in the bunching process, for example, from nonlinearities in the RF fields, cause 


\section{DISCLAIMER}

This report was prepared as an account of work sponsored by an agency of the United States Government. Neither the United States Government nor any agency thereof, nor any of their employees, make any warranty, express or implied, or assumes any legal liability or responsibility for the accuracy, completeness, or usefulness of any information, apparatus, product, or process disclosed, or represents that its use would not infringe privately owned rights. Reference herein to any specific commercial product, process, or service by trade name, trademark, manufacturer, or otherwise does not necessarily constitute or imply its endorsement, recommendation, or favoring by the United States Government or any agency thereof. The views and opinions of authors expressed herein do not necessarily state or reflect those of the United States Government or any agency thereof. 


\section{DISCLAMIER}

Portions of this document may be illegible in electronic image products. Images are produced from the best available original doccument. 

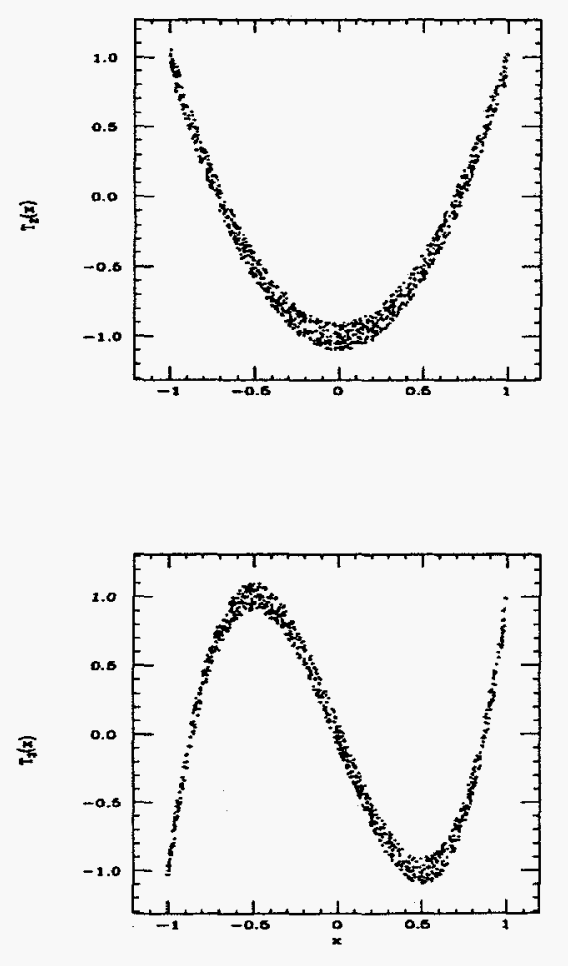

Figure 1: Mode patterns for $n=2$ and $n=3 ; T_{1}(x)=x$.
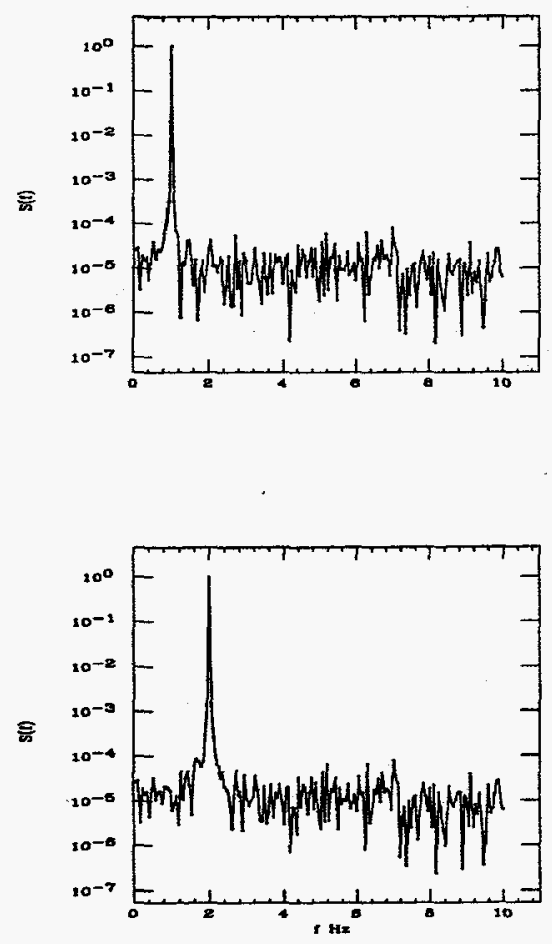

Figure 2: Modulation spectra for $n=1$ fundamental and $n=2$ second harmonic.

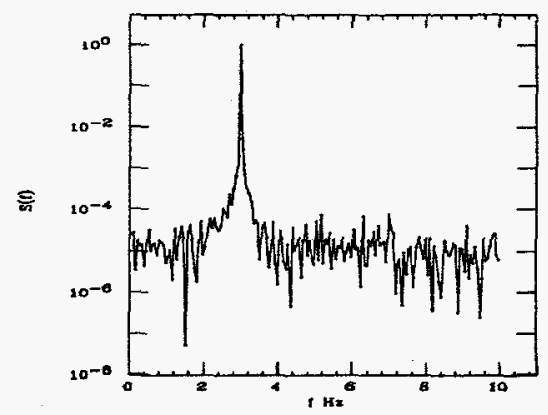

Figure 3: Modulation spectrum for $n=3$.

this best case limit to seldom be achieved. Having a means to quantify and correct the bunch length is clearly useful for speeding and automating the optimization process. Tschebyshev analysis of the phase-phase transfer map $\left(M_{55}\right)$ provides a systematic means of quantifying the nonlinearities as the amplitude of certain modes in phase-phase space. Given the measured amplitudes, it is clear that the bunch length is made smaller to the extent that the individual amplitudes can be made smaller.

In terms of the Tschebyshev modes, the $n=1$ mode gives the slope of the phase transfer function. Making the $n=1$ term in the expansion zero $\left(a_{1}=0\right)$, is equivalent to the demand that the bunch should be going through a longitudinal waste $\left(\sigma_{55}=0\right)$. Demanding that $a_{2}=0$, means that bunch particles starting at the front of the bunch, the back of the bunch, and the middle of the bunch all arrive at the same time, given that $a_{1}$ has previously been corrected and the higher order terms are smaller than these. In this case the nonlinear transfer matrix element $T_{555}$ is zero for the bunching system. Setting $a_{3}$ to zero, provides the first term that can correct asymmetries between the front and back of the bunch.

The level of correction that has been achieved in the references can be categorized. For example, Dowell, et. al., [2] and Carlsten [3] have corrected the $n=1$ and $n=2$ modes. Likewise, Krafft [1] has corrected through $n=3$, at least for the low space charge case reported.

\section{EXPERIMENTAL RESULTS}

At Jefferson Lab, for many years it has been possible to measure the phase-phase correlation function $\left(M_{55}\right)$ between the beam chopping system in the injector and strategically placed longitudinal pickup cavities in the injector. An illustrative case is presented in Figures 4 and 5. In Fig. 4 an experimental aquisition of a 600 point phasephase correlation function is given by the unconnected dots. Each dot was obtained on a separate beam pulse and the modulation of the input phase was chosen to be in a triangle wave so that uniform filling of the display is obtained [4]. 
Next the modulation was changed to be fully sinusoidal, all other conditions remaining the same. By the above analysis, the correlation function should be resolved into its Tschebyschev modes by examining the Fourier amplitudes of the output phase modulation. In Fig. 5, a Fourier analysis of the output phase is given which clearly shows the correct harmonic structure. The modulation frequency was $65 \mathrm{~Hz}$, which is aliased to $5 \mathrm{~Hz}$ with a $60 \mathrm{~Hz}$ sample rate, and the total number of points was $1020(17 \mathrm{sec})$. The resulting Tschebyshev expansion coefficients are shown in Table 1. Fig. 4 shows the function that results when the modes up to $n=5$ are synthesized. The expansion clearly reproduces the measured phase-phase correlation function.

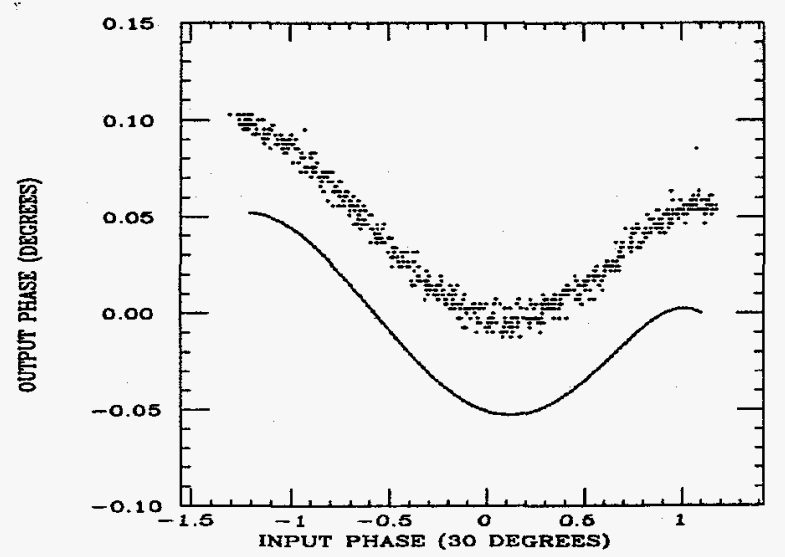

Figure 4: Experimental phase-phase transfer function obtained at the Jefferson Lab injector, and its synthesis using the Tschebyshev expansion (displaced for comparison).

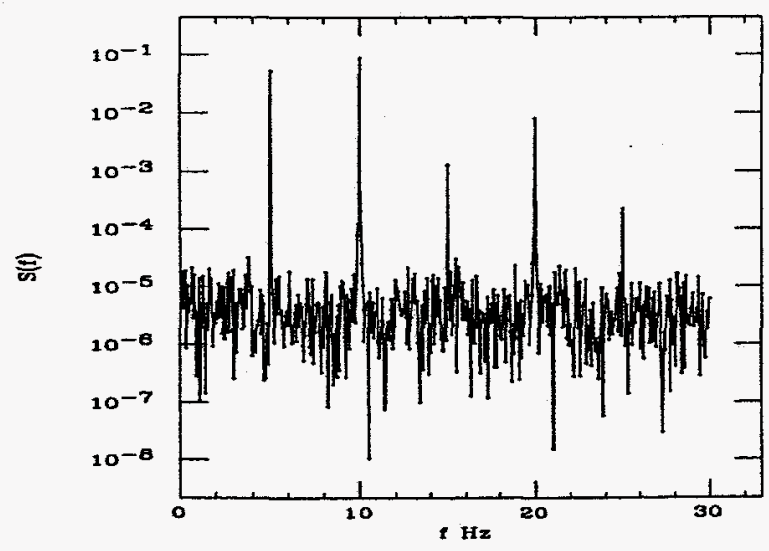

Figure 5: Output phase spectrum, $f=65 \mathrm{~Hz}$.

Because as part of the measurement process the power

\begin{tabular}{|c|c|}
\hline$n$ & $a_{n}\left(^{\circ}\right)$ \\
\hline 1 & -0.458 \\
2 & 0.599 \\
3 & 0.072 \\
4 & -0.182 \\
5 & -0.031 \\
\hline
\end{tabular}

Table 1: Expansion coefficients for phase-phase transfer function in Fig. 4.

spectrum is obtained, one can estimate the relative error of the measurements. Clearly, the peak at $n=5$ can be distinguished with a $S / N$ about 10 in Fig. 5. As the expansion coefficient is $a_{5}=-0.031^{\circ}$, the distortion in arrival time cleanly measured is $a_{5} * 668 \mathrm{psec} / 360^{\circ}$, or 58 fsec. It is possible to discern modulations perhaps onefifth intense, leading to an overall resolution of order 25 fsec. This experimental result has precision comparable to the optical techniques that have been used to determine bunch length, but the present measurement provides much more information which can be used to tune the bunch length, in that the types of distortion in the phase space are given directly.

\section{CONCLUSIONS}

The conclusions of this work are:

- Nonlinear transfer maps may be conveniently analysed experimentally by performing modulation measurements, and by quantifying the results obtained using Tschebyshev polynomials.

- The nonlinearity is minimized when the magnitude of the expansion coefficients is minimized.

- Measuring the phase-phase correlation function provides a useful means of obtaining data to minimize the bunch length.

It should be noted that the Tschebyshev expansion techniques mentioned above are useful in quantifying measurements of transverse beam optical nonlinearities using transverse modulation of the beam. Multiple dimensions may be analyzed in the same way using multiple modulation frequencies. This work supported by U. S. DOE Contract No. DE-AC05-84ER40150.

\section{REFERENCES}

[1] Krafft, G. A., Proc. of the Microbunches Workshop, AIP Conf. Proceedings No. 367, pgs. 46-55 (1995).

[2] Dowell, D. H., Hayward, T. D., and Vetter, A. M., Proc. 1995 Particle Accelerator Conference, pgs. 992-994 (1995).

[3] Caristen, B. E., Proc. of the Microbunches Workshop, AIP Conf. Proceedings No. 367, pgs. 21-35 (1995).

[4] Bowling, B. A., et. al., "The CEBAF Injector Phase Transfer Function Measurement System", in preparation. 